\title{
PERSPECTIVA AMBIENTAL DE LAS COCINAS SOLARES EN LA ZONA ALTOANDINA DE TACNA
}

Hugo Torres Muro ${ }^{1}$; Carlos Polo Bravo ${ }^{2}$ Boris Milla Taco

\begin{abstract}
$R E S U M E N$
La población altoandina de Tacna, en su gran mayoria, usa leña, bosta y yareta como fuentes de energia, para cocinar sus alimentos; esto ocasiona emisión de $\mathrm{CO}_{2}$ por combustión, contaminación atmosférica y sus consecuencias globales. El trabajo de investigación tiene como objetivo determinar el impacto ambiental que produciria el uso de Cocinas Solares en la zona objetivo. Desde el punto de vista de la perspectiva ambiental que tienen las cocinas solares, los resultados obtenidos nos permiten concluir que su aplicación masiva es una alternativa de solución para la zona altoandina de Tacna, que permitirá revertir la situación actual causando impactos positivos significativos, como la reducción de la deforestación, reducción de la migración y extinción de especies, disminución de enfermedades respiratorias, reducción de la degradación de los suelos, mejoras en la calidad de la dieta alimenticia y por lo tanto una mejor calidad de vida de la población.
\end{abstract}

\section{A BSTRACT}

The population altoandina of Tacna, in her great majority, uses firewood, dung and yareta like energy sources, to cook her allowances; this causes emission of $\mathrm{CO}_{2}$ for combustion, air pollution and her global consequences. The investigation work has as objective to determine the environmental impact that would produce the use of solar cookers in the area objective. From the point of view of the environmental perspective that have the solar cookers, the obtained results allow us to conclude that their massive application is a solution alternative for the area altoandina of Tacna that will allow to revert the current situation causing significant positive impacts, as the reduction of the deforestation, reduction of the migration and extinction of species, decrease of breathing illnesses, reduction of the degradation of the floors, improvements in the quality of the nutritious diet and therefore a better quality of the population's life.

\section{INTRODUCCIÓN}

En las zonas rurales del Perú, el uso de la leña como fuente primaria de energía representa una fracción muy importante de todas las necesidades energéticas de la población y su escasez es cubierta en muchos casos por el uso de la bosta y la yareta. La población altoandina de Tacna, en su gran mayoría usan la leña, la bosta y la yareta como fuentes primarias de energía, para múltiples aplicaciones domésticas y artesanales como la preparación de sus alimentos y el teñido de Iana; esto ocasiona emisión de $\mathrm{CO}_{2}$ por combustión, exacerbando la contaminación atmosférica y sus consecuencias globales.

Esta práctica ancestrai, produce también una fuerte presión sobre los bosques de Queñua, particularmente en las provincias de Tarata y Candarave. Esto se agrava por la baja eficiencia térmica de estas cocinas lo que significa mayor demanda de combustible y por lo tanto mayor deforestación. La deforestación y desertización por la tala indiscriminada de bosques produce pérdic de ecosistemas y extinción de algunas especies.

El uso de la bosta como fuente de energía primar produce una pérdida de fertilidad de las tierras en don se encuentran ubicados los pastos naturales que sirv de alimento a los camélidos sudamericanos y otr tipos de ganadería, que constituyen la principal fuer de ingresos económicos de las comunidad involucradas. La quema de estiércol agota la fertilid de la tierra, baja el rendimiento de las cosechas contribuye al esparcimiento del hambre, la migraci de la gente y de los rebaños a tierra más habitable ciudades ya sobre pobladas.

La reforestación, el uso de fuentes renovables energía y la conservación de combustibles, son tres métodos que ofrecen soluciones posibles a crisis global de la energía en general y a la crisis d leña en particular. En la actualidad se ha consegu una solución tecnológica para que en una media

(1) Lic. en Fisica

(2) Lic. en Fisica / Energia Solar

(3) Bach. en Fisica Aplicada 
250 dias al año la cocción de alimentos sea limpia y sin malgastar leña. Nos referimos a la cocina solar. Existen diferentes tecnologías, pero en todas ellas se obtienen temperaturas idóneas para la cocción saludable de los alimentos. La energia solar como fuente de energía que sustituya a la leña, representa un aporte importante en los programas para combatir la deforestación y desertificación.

\section{MATERIALES Y MÉTODOLOGIA}

\subsection{ACTIVIDADES REALIZADAS}

- Visita de inspección a los lugares afectados por la extracción de leña.

- Encuestas para determinar el perfil de extracción, consumo y comercialización de leña.

Estimación de la cantidad de $\mathrm{CO}_{2}$ que se emite a la atmósfera por el uso de la leña en la zona de estudio.

Evaluación de la eficiencia térmica de la cocina solar en la zona de estudio.

Estimación de la cantidad de $\mathrm{CO}_{2}$ que se dejaría de emitir con el uso de cocinas solares parabólicas SK14.

- Demostraciones del manejo y mantenimiento de la cocina, preparación de alimentos, tiempo de cocción. etc.

\subsection{ESTIMACIÓN DE LA EMISIÓN DE $\mathrm{CO}_{2}$ PRODUCIDO POR EL USO DE LEÑA}

En la determinación de la demanda de leña, como fuente de energía primaria para aplicaciones domésticas y artesanales en la zona altoandina de Tacna, provincia de Tarata a partir de las encuestas realizadas, se han obtenido los siguientes resultados,

* Cantidad que consumen por mes/familia (1 carga es igual a $40 \mathrm{Kg}$ ): $320 \mathrm{~kg}$

* Total de leña que se consume por mes (250 familias): $80000 \mathrm{~kg}$

* Total de leña que se consume por año (250 familias): $960000 \mathrm{~kg}$

* Costo de la leña: $\mathrm{S} /, 0,50 \mathrm{~kg}$

* Costo de leña por mes: S/. 40000

* Costo de leña por año: S/. 480000

* Comercialización: El 30 \% compran leña y un 70 $\%$ consigue su propia leña.

* Existen pocos intermediarios.

* Los tiempos promedios que utilizan para conseguir la leña son entre 3 a 6 horas, por que los lugares de extracción cada vez están más lejos del pueblo, a más de $10 \mathrm{~km}$.

Si considerando que 01 tonelada de leña al ser quemada produce 1,83 toneladas de $\mathrm{CO}_{2}$, podemos estimar la cantidad de $\mathrm{CO}_{2}$ que se está emitiendo a la atmósfera en la provincia de Tarata, teniendo en cuenta la demanda de leña calculada a partir de las encuestas equivalentes a $960 \mathrm{~T} / \mathrm{a}$, entonces obtenemos que la cantidad de $\mathrm{CO}_{2}$ emitida por el uso de la leña para cocinar alimentos en la zona de estudio es de 1756,8 T/a.

\subsection{ESTIMACIÓN DE LA CANTIDAD DE CO2 QUE SE DEJA DE EMITIR POREL USO DE COCINAS SOLARES}

Para estimar las cantidades de $\mathrm{CO}_{2}$ que se dejarán de emitir a la atmósfera por el uso de las cocinas solares parabólicas, en la zona alto andina de Tacna se debe considerar según el Ing. Dieter Seifert (1998), lo siguiente:

$>$ Datos de la Cocina Solar tipo SK12, (SK14, SK98): Diámetro, $\mathrm{D}=1,4 \mathrm{~m}$; Eficiencia, $\mathrm{h}=50 \%$

> Energia Solar máxima anual a la Sk12 continuamente orientada al sol: $E_{\max }=3110 \mathrm{kWh} / \mathrm{m}^{2} / \mathrm{a}$

Apertura A de la cocina SK12, con seguimiento, latitud $18^{\circ}$ es $1,5 \mathrm{~m}^{2}$

$>$ Energia efectiva de una cocina SK12: $E_{n}=E_{\max } A$ $\mathrm{h}=2333 \mathrm{kWh} / \mathrm{a}$

> Poder Calorifico de la leña: $H=15000 \mathrm{~kJ} / \mathrm{kg}=$ $4,17 \mathrm{kWh} / \mathrm{kg}$

$>$ Rendimiento del fuego a tres piedras: ? $=0,05$

$>$ Energía efectiva de $1 \mathrm{~kg}$. de leña: $E_{n 1}=H$ ? = $0,21 \mathrm{kWh} / \mathrm{kg}$

$>$ Equivalencia máxima de leña por SK12: $m_{\max }=$ $E_{\max } / E_{n 1}=11196 \mathrm{~kg} / \mathrm{a}$

- Emisión de $C O$ de la equivalencia máxima: $M_{\max }$. $\mathrm{CO} 2=m_{\max }(1,83)^{2}=20488,68 \mathrm{~kg}$ de $\mathrm{CO}$ laño (considerando que $4 \mathrm{~T}$ de leña emite a la atmôsfera 7,32 T de CO2).

La cantidad máxima de $\mathrm{CO}_{2}$ que se dejará de emitir a la atmósfera con el uso de una cocina solar SK12 continuamente orientada, sustituyendo al fuego de tres piedras es: $M_{\text {max }}=20488,68 \mathrm{~kg} / \mathrm{a}$

La cantidad de $\mathrm{CO}_{2}$ que se puede dejar de emitir a la atmósfera en forma directa con el uso de una cocina solar SK12 común suponiendo un uso de 2000 horas por año (10 h/d durante 200 dias del año) se obtiene con los siguientes parámetros:

- Temporada T del uso anual: 2000 h/a 
- Potencia N de la cocina solar SK12: 0,6 kW

- Energía solar máxima para un tiempo de $10 \mathrm{~h} / \mathrm{d}$ expuestas al sol (de $8 \mathrm{~h}$ a $17 \mathrm{~h}$ ): $\mathrm{E}_{\max 2}=1600 \mathrm{kWh} /$ $\mathrm{m}^{2} \mathrm{a}$

- Energía efectiva de la cocina SK12: $E_{n 2}=E_{\max 2}$ $\mathrm{A} \eta=1200 \mathrm{kWh} / \mathrm{a}$

- Equivalencia de leña por SK12: $m_{2}=E_{n 2} / E_{n 1}=$ $5714,3 \mathrm{~kg} / \mathrm{a}$

W La cantidad de $\mathrm{CO}_{2}$ que se puede dejar de emitir con una cocina solar parabólica SK12 común, expuesta $10 \mathrm{~h} / \mathrm{d}$ al sol es: $m_{2}$. CO $=m_{2} 1,83=10456,6 \mathrm{~kg}$ de $\mathrm{CO} / \mathrm{a}$

Extendiendo estos resultados para el caso de Tarata, en dónde existe una población estimada de 1250 habitantes para el año 2000 (según datos del INEI) y considerando que cada familia está conformada por 5 personas, se tiene que la cantidad máxima de $\mathrm{CO}_{2}$ que se puede dejar de emitir a la atmósfera con el uso de cocinas solares SK12 por 250 familias de Tarata es de $2614,2 \mathrm{~T} / \mathrm{a}$

\subsection{IDENTIFICACIÓN Y VALORACIÓN CUALITATIVA DE IMPACTOS AMBIENTALES}

En la Identificación de impactos ambientales se ha considerado los impactos producidos por la extracción de la leña, por el uso de la leña para cocinar, y los posibles impactos ambientales que producirán el uso de las cocinas solares parabólicas SK12. En los diagramas de redes que se mostrarán a continuación hay que considerar que el color rojo indica un impacto negativo importante, el color amarillo indica un impacto negativo moderado y el color verde indica un impacto positivo importante y el color celeste un impacto positivo moderado. Los números romanos que aparecen en los diagramas de redes indican lo siguiente: I Alteraciones en el Medio Físico; II Alteraciones en el Medio Biótico y III Alteraciones en el medio Socio Cultural.

\section{RESULTADOS}

- La demanda de leña calculada a partir de las encuestas asciende a $960 \mathrm{~T} / \mathrm{a}$

- La cantidad de $\mathrm{CO}_{2}$ que se está emitiendo a la atmósfera por el uso de la leña para cocinar es de $1756,8 \mathrm{~T} / \mathrm{a}$.

- La cantidad máxima de $\mathrm{CO}_{2}$ que se dejará de emitir a la atmósfera con el uso de una cocina solar SK12(SK14), continuamente orientada, sustituyendo al el fuego de tres piedras es: $M_{\max }=20488,68 \mathrm{~kg} / \mathrm{a}$

- La cantidad de CO que se puede dejar de emitir con una cocina solar parábólica SK12 común, expuesta $10 \mathrm{~h} / \mathrm{d}$ al sol es: $m_{2}$. CO $=m_{2} 1,83=10456,6 \mathrm{~kg}$ de $\mathrm{CO} / \mathrm{a}$.
- La cantidad de CO que se puede dejar de emitir a la atmósfera con el úso de cocinas solares SK12 por 250 familias de Tarata es de 2614,2 T/a.

\section{DIAGRAMA DE REDES DE IMPACTOS} AMBIENTALES EN LA EXTRACCIÓN DE LEÑA EN LAZONAALTOANDINA DE TACNA

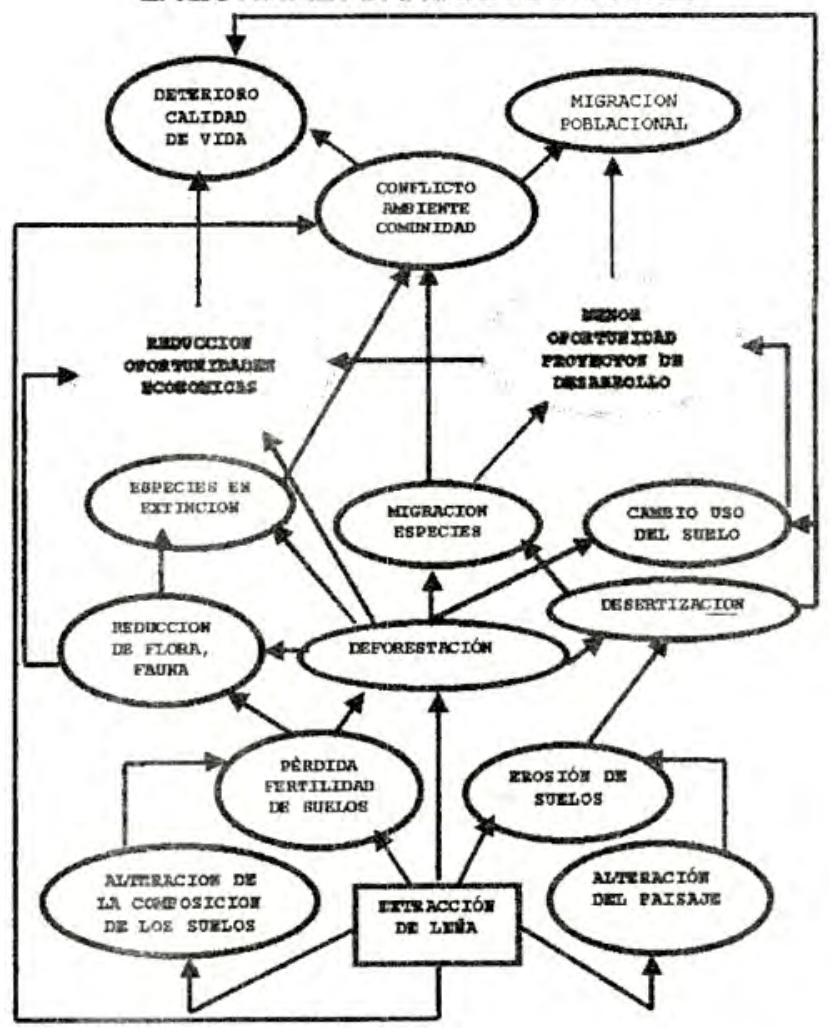

DIAGRAMADE REDES DE IMPACTOS AMBIENTALES DEL USO DE LA LENA PARA COCINAR EN LAZONAALTOANDINA DE TACNA

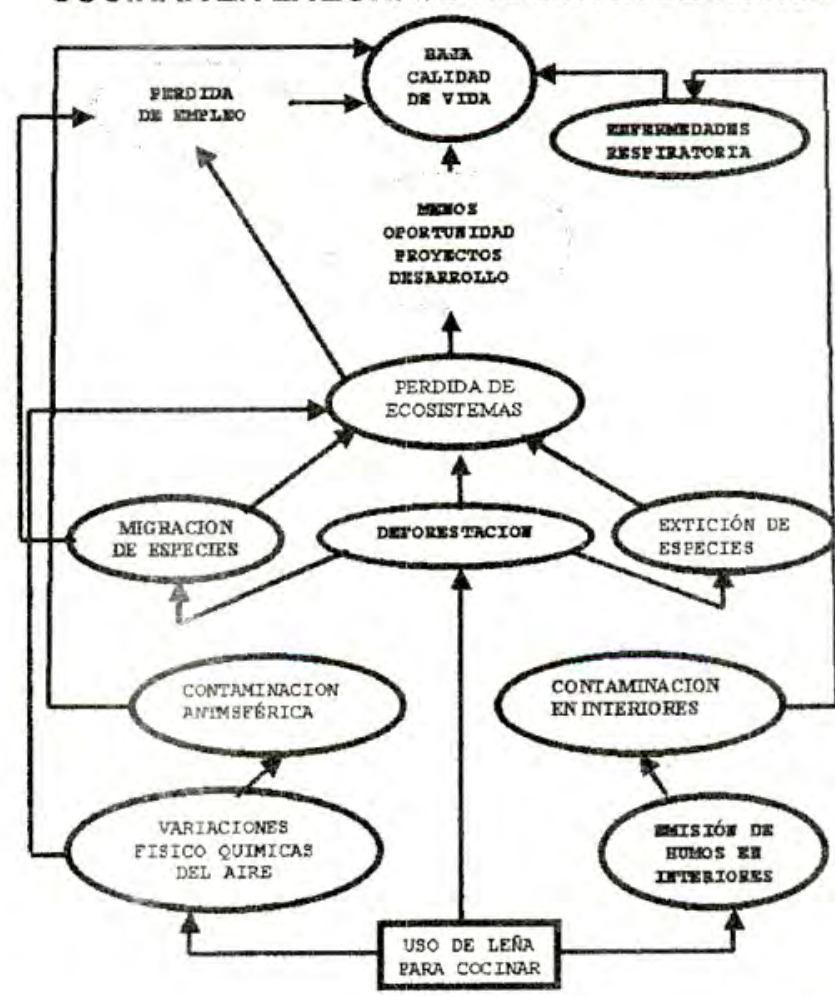




\section{DIAGRAMA DE REDES REDUCIDA DE POSIBLES IMPACTOS AMBIENTALES PRODUCIDO POR EL USO DE COCINAS SOLARES EN LAZONA ALTOANDINATACNA}

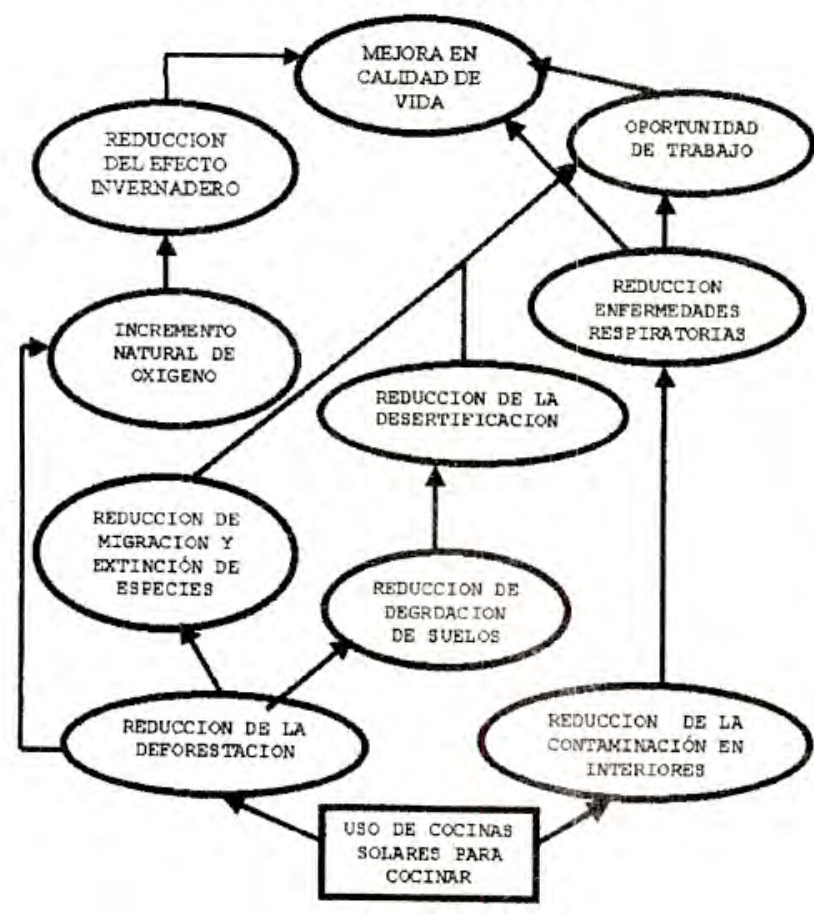

\section{CONCLUSIONES}

- La fuerte demanda de leña esta causando una serie de impactos ambientales, económicos y sociales en la población de la zona de estudio, que contribuye a incrementar los bajos niveles de calidad de vida de las comunidades en estudio.

- Los impactos ambientales más importantes son sobre los ecosistemas, flora, fauna lo cual está provocando que exista una creciente migración y extinción de especies, asi como una paulatina pero constante degradación de los suelos por erosión causada por las lluvias principalmente.

- El uso de leña para cocinar sus alimentos causa impactos negativos importantes en la población, sobre todo en aquellas personas que se encuentran la mayor parte del tiempo en la cocina, esto deberá ser verificado a través de un estudio epidemiológico detallado.

- La introducción de cocinas solares parabólicas SK12, SK14 o SK98 pueden ser una alternativa de solución para solucionar la crisis de la leña que está ocurriendo en la zona altoandina de Tacna, permitirá revertir la situación actual y además causará impacto positivos considerables, como la reducción de la deforestación, reducción de la migración y extinción de especies, reducción de enfermedades respiratorias, reducción de la degradación de los suelos, mejoras en la calidad de la dieta alimenticia y por lo tanto una mejor calidad de vida de la población.
- Es necesario realizar un estudio detallado de los impactos que sobre las especies de flora y fauna tiene la extracción de la leña, particularmente de la Queñua, en la zona altoandina de Tacna.

-Es necesario que las municipalidades y los gobiernos regionales consideren en sus planes estratégicos de desarrollo la implementación de un programa de capacitación y difusión para el uso masivo de las cocinas solares en la zona altoandina de Tacna, no sólo de las cocinas parabólicas SK sino también de las cocinas solares tipo caja y de las cocinas mejoradas, de acuerdo al la aceptación que este tipo de tecnologia tenga en dichas comunidades.

\section{REFERENCIA BIBLIOGRÁFICA}

1. Aguilar, Margot, (1982), Cocinando con energía solar para cuidar la Tierra, Ediciones GEA, Mexico D.F.

2. Canter, L. (1998), Manual de Evaluación de Impacto Ambiental, Mc. Graw Hill, 2da Edición, Madricl.

3. CEPIS-OPS/OMS, (1999), Evaluación de Impacto Ambiental, http//www. cepis ops-oms.org

4. Conesa Fdez, V. (1997), Guía Metodológica para la Evaluación de impacto Ambiental, Ediciones Mundi Prensa, Madrid.

5. Finck Pastrana, A. Et al. (2001), Correcciones al protocolo de ensayos de cocinas solares para la determinación del rendimiento térmico, www.g.unsa.edu.ar/asades/actas $2000 /$ 03-81. html.

6. Finck Pastrana, A., (2001), Protocolo de cocción solar de RICSA apreciaciones respecto de la determinación de la energía y la carga para determinar la potencia efectiva de cocción, www.g.unsa.edu.ar/asades/actas2000/03-81.html,

7. Fundación TIERRA, (2000), Perspectiva Ambiental 25: La Cocina Solar, Barcelona, España.

8. IDMA, (1998), Estudio de Demanda de Leña y su Impacto Ambiental en el Santuario Nacional de Ampay, Apurimac, Perú.

9. Mullick, S.C. Et. Al. (1991), Thermal Test Procedure for a Paraboloid Concentrator Solar Cooker, Solar Energy, vol 46, USA.

10. Nandwani S., Et. al. (2000), Horno/cocina solar: Construcción y financiamiento, II Worid Conference On Solar Cookees.

11. ONU - Para la agricultura y la alimentación, (1979) Sistemas de almacenamiento de energía para cocinar en las zonas rurales en desarrollo, Lawland

12. Torres Muro, Hugo; Polo Bravo, Carlos; López Cornejo, Orlando (2002), Impacto de las Cocinas Solares sobre la demanda de leña y la emisión de Bióxido de Carbono en la zona Altoandina de Tacna, CERT, UNJBG, Tacna.

13. Seifert D. y Vilchez M., (1999), La cocina solar SK98, $4^{\circ}$ ENCUENTRO SOLAR, Benicarlo, 26 y 27 de junio de 1999

14. Seifert, D. (1996), Solar Cooker SK12 - Experiences and Visions. En: Procesdings EuroSun'96, 10. Internationales Sonnenforum, voi. 4 , p. $1.483-1.487$ 


\section{ANEXO}

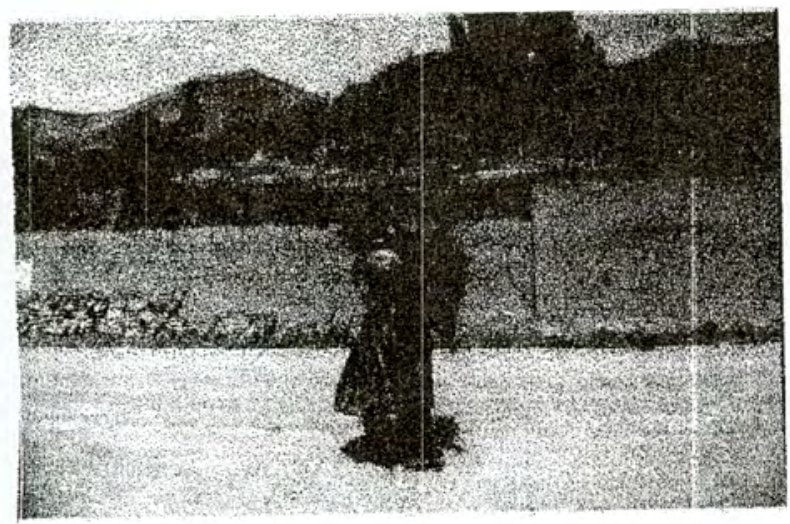

Foto 1: Vista que muestra la forma cómo las mujeres contribuyen con la recolección de la leña, sin utilizar animales de carga

Foto 2: Estudiantes y profesores evaluando y difundiendo el uso de las cocinas solares y otros sistemas solares, en la ciudad de Tarata.
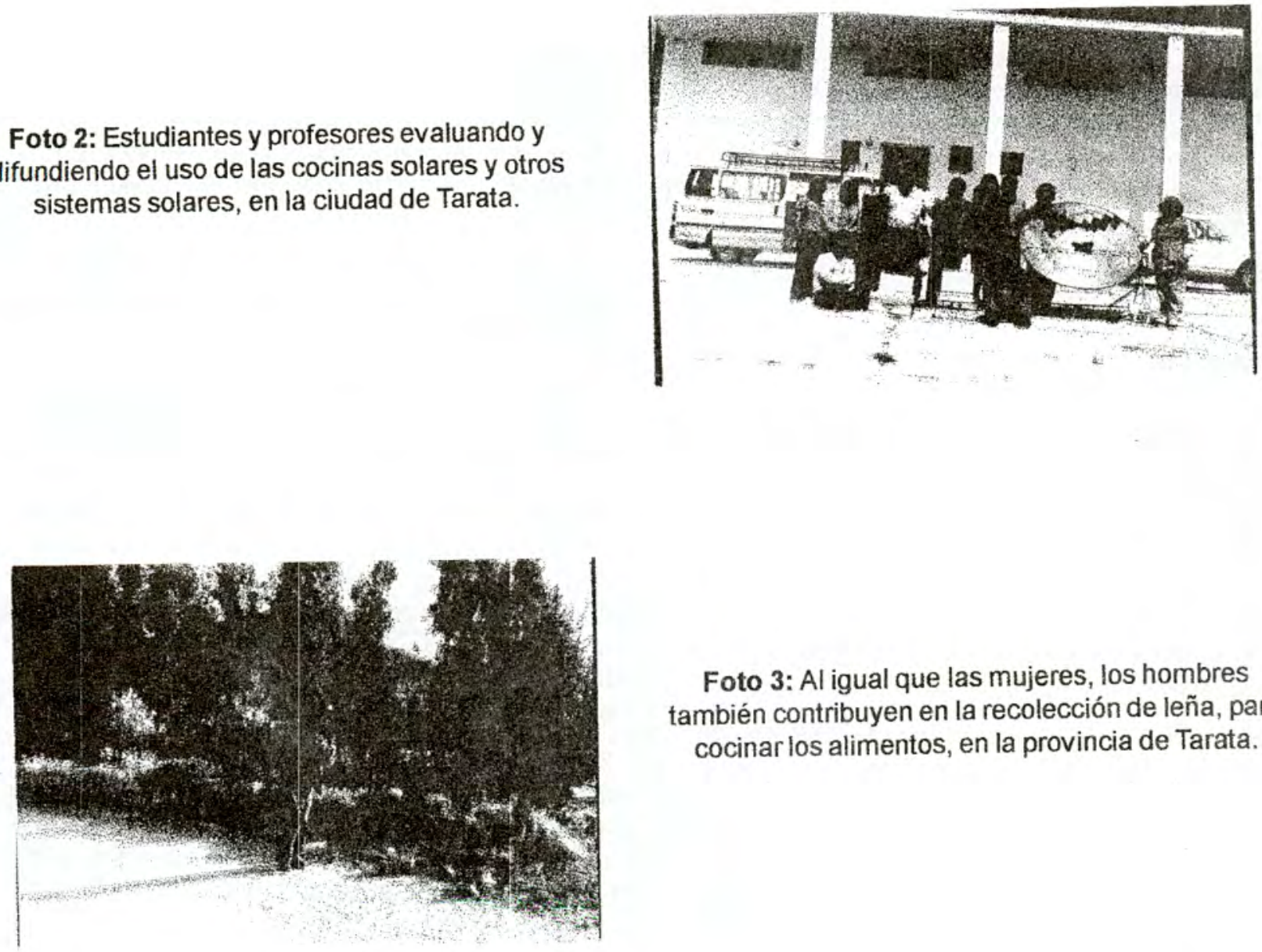

Foto 3: Al igual que las mujeres, los hombres también contribuyen en la recolección de leña, para cocinar los alimentos, en la provincia de Tarata. 